\title{
Vigilancia de infecciones nosocomiales en un hospital de cardiología
}

\author{
Julio D. Molina-Gamboa, M.C., ${ }^{(1)}$ Héctor Garza-Moreno, Enf. en S.P. ${ }^{(1)}$
}

\section{Molina-Gamboa JD, Garza-Moreno H. Vigilancia de infecciones nosocomiales en un hospital de cardiología. Salud Publica Mex 1999;41 suppl 1:S26-S31.}

\begin{abstract}
Resumen
Objtivo. D escribir los resultados de la vigilancia de las infecciones nosocomiales en un hospital de cardiología y neumología del norte de México en sus primeros meses de actividad. Material y métodos. Informe retrospectivo de los hallazgos de la vigilancia de infecciones nosocomiales realizado por el equipo de control, con búsqueda directa de la información mediante la revisión de pacientes, expedientes y notas médicas y de enfermería, reportes de bacteriología y reportes del personal encargado de la atención de los enfermos. Las tasas de infección se dividieron en generales y específicas y se calcularon de la manera habitual, con análisis de los resultados por prueba de $c^{2}$ y t de Student. Resultados. La tasa promedio de infección durante el periodo fue de 4.99, aunque fluctuó significativamente durante los periodos vacacionales del personal del equipo de control. Por frecuencia, las infecciones más comunes fueron las de la herida quirúrgica (1.14), las de vías urinarias (1.08) y las bacteriemias primarias (0.72). Sin embargo, al agrupar estas últimas con el resto de las infecciones asociadas a catéteres, estas mismas infecciones fueron las más frecuentes (1.73). Los gérmenes más co múnmente encontrados fueron grampositivos, aunque en el caso de las neumonías la frecuencia mayor la ocuparon los gramnegativos y, en el caso de las infecciones urinarias, la Candida spp. La mortalidad aso ciada a infecciones no socomiales alcanzó $21.7 \%$, y la tercera parte de estos fallecimientos estuvo directamente relacionada con la infección. A simismo la estancia hospitalaria y los costos se elevaron significativamente con la presencia de infecciones noso comiales. Conclusiones. Las infecciones nosocomiales son comunes en hospitales de cardiología intervencionista y elevan significativamente la mo rtalidad y el costo de la atención de los pacientes que sufren padecimientos cardiotorácicos. Se requiere del establecimiento de un sistema de vigilancia eficaz que elimine al mí-
\end{abstract}

Molina-Gamboa JD, Garza-Moreno H. Nosocomial infections surveillance in a cardiology and thoracic diseases hospital. Salud Publica Mex 1999;41 suppl 1:S26-S31.

\begin{abstract}
A bstract
Objective. To describe a nosocomial infection surveillance in a cardiology and thoracic diseases hospital in N orthern Mexico during its initial months of activity. Material and methods. Restrospective report of nosocomial infection surveillance performed by the infection control team with patient visits, clinical records, microbiology reports, and direct information obtained by health personnel. General and specific infection rates were analized by the $c^{2}$ test and the Student $t$ test. Results. General infection rate was 4.99 during the 17 month period. Monthly infection rates changed significantly during infection control team vacations. By site of infection, surgical wound infection were the most common (1.14), followed by urinary tract infections (1.08), and bacteremia (0.72). However, when we grouped bacteremias and other cateter related infections, this intravascular access related infections were the most frequent (1.73). Gram positive organisms were most commonly isolated in nosocomial infections, except in pneumonias (gram negative organism) and urinary tract infections (Candida spp.). Mortality related to nosocomial infection was high (21.7\%), and at least one third of those deaths was directly related to the infection. In hospital stay and cost were also increase with nosocomial infections. Conclusions. N osocomial infection is common in interventional cardiology hospitals, producing high mortality and rising costs and length of stay. We need a more efective nosocomial infections surveillance system to eliminate changes in monthly case detection produced by the infection control team vacations, since this underregistry could result in lost of control of nosocomial infections and epidemic outbreak emergence.
\end{abstract}

(1) Hospital de Enfermedades Cardiovasculares y del Tórax N 0. 34, IMSS, Monterrey, N uevo León, México.

Fecha de recibido: 19 de febrero de 1998 - Fecha de aprobado: 6 de agosto de 1998

Solicitud de sobretiros: Dr. Julio D. Molina. Hospital de Enfermedades Cardiovasculares y del Torax N 0. 34. Servicio de Infectología. Lincoln y Av. Patrimonio Familiar, colonia Valle Verde, Primer Sector, Monterrey, N uevo León, México. 
nimo las fluctuaciones en la captación de los casos durante el periodo vacacional del personal del equipo de control de infecciones, ya que el subregistro de los casos puede traer como consecuencia la pérdida de control so bre las infecciones hospitalarias y la aparición de brotes epidémicos.

Palabras clave: infección hospitalaria; control de infecciones; hospitales con 300 a 499 camas; México
Key words: cross infection; infection control; hospital bed capacity, 300 to 499; Mexico
A pesar del interés que ha surgido recientemente en México respecto a las infecciones intrahospitalarias, ${ }^{1}$ la mayoría de las acciones en esta área se han enfocado a los hospitales generales o a aquellos en los cuales se considera que existe mayor riesgo de infección (hospitales de hemato-oncología, pediatría, centros de trasplante, etc.). En el área de cardiología, sin embargo, es poco lo que se sabe acerca del impacto de las infecciones intrahospitalarias en la morbilidad y la mortalidad de los pacientes, así como de los costos que éstas implican. En el informe de Ponce de León y colaboradores ${ }^{2}$ se comentan los datos obtenidos tras la vigilancia, por cuatro meses, de infecciones nosocomiales en el Instituto Nacional de Cardiología, en donde se obtuvo una tasa de infecciones de $17 \%$, si bien no se especifica la forma en que se detectaron y los tipos más frecuentes en ese hospital. En un informe del Hospital for Sick Children de Toronto, Canadá, la tasa de incidencia de infecciones en cirugía pediátrica cardiovascular fue de $25.2 \%{ }^{3}$ Estas cifras reflejan la importancia que pueden tener las infecciones nosocomiales para los pacientes sometidos a procedimientos diagnósticos y terapéuticos avanzados en el área de cardiología.

Al inaugurarse, al final de 1995, el Centro Médico Nacional del Noreste de Enfermedades Cardiovasculares y del Tórax (CMNNECT) del Instituto Mexicano del Seguro Social (IMSS) -ahora llamado Hospital de Enfermedades Cardiovasculares y del Torax No. 34 (HECVT 34)-, destinado básicamente a la práctica de la cardiología y de la cirugía cardiovascular, una de las grandes preocupaciones consistió en contar con un sistema de vigilancia y control de las infecciones intrahospitalarias que redujera al mínimo los problemas y los costos que ocasionan. En consecuencia, se optó por trabajar con personal altamente capacitado y dedicado en forma exclusiva a esa actividad. Esa situación no es característica de otros hospitales del IMSS, donde una misma persona desempeña todas las actividades propias de la medicina preventiva: vacunación, notificación de enfermedades transmisibles, de defunciones, etcétera, además de la vigilancia de las infecciones nosocomiales.
El objetivo del presente estudio ha sido reconocer la frecuencia con la que ocurren las infecciones intrahospitalarias en México, y determinar los factores de riesgo relacionados con las mismas, así como la mortalidad y el costo que acarrean estas complicaciones.

\section{Material y métodos}

El HECVT 34 es un hospital de tercer nivel donde se atienden pacientes adultos y pediátricos de la región noreste de México (Chihuahua, Durango, Zacatecas, Coahuila, Tamaulipas, San Luis Potosí y Nuevo León) que requieren atención especializada en cardiología, cirugía cardiovascular y torácica, neumología y angiología. Cuenta con 300 camas censables, cinco unidades de terapia intensiva (coronaria, neonatal, respiratoria, posquirúrgicas de adultos y niños) y seis quirófanos, donde cada día se llevan a cabo un promedio de siete cirugías, la mayoría a corazón abierto.

El equipo de control de infecciones está conformado por el infectólogo, en su calidad de coordinador general, y un enfermero epidemiólogo, encargado de la vigilancia diaria. La mayoría de las detecciones se realizaron de manera directa, y fueron pocos los casos notificados por el personal encargado de la atención directa de los pacientes en los diferentes servicios. La búsqueda de casos se realizó también mediante los expedientes clínicos y los informes de bacteriología. Todos aquellos pacientes con sospecha de infección fueron revisados por el coordinador antes de incluirlos como casos de infección intrahospitalaria.

Se utilizaron los criterios previamente establecidos por el Centro para la Prevención y el Control de las Enfermedades de Estados Unidos de América (EUA) para definir la infección nosocomial. ${ }^{4}$ Sólo se consideró como infección nosocomial aquella ocurrida después de 72 horas del ingreso del paciente.

La tasa de infecciones nosocomiales se calculó con base en el número de episodios de infección captados, dividido entre el número de egresos y multiplicando el resultado por 100. La tasa de incidencia se calculó dividiendo el número de pacientes infectados entre el 
número de egresos, por 100. La tasa de infección relacionada con procedimientos específicos ${ }^{5}$ (instalación de sonda urinaria, intubación endotraqueal, colocación de catéter central) se calculó dividiendo el número de infecciones asociadas al procedimiento específico entre el número de procedimientos realizados en el mismo periodo de tiempo, por 100. La tasa de infecciones por dispositivos utilizados (sondas urinarias, ventilación mecánica, uso de catéter central) se calculó dividiendo el número de infecciones asociadas al uso de dicho dispositivo, entre el número de días que se mantenía dicho dispositivo en uso, por 100.

El estudio se llevó a cabo entre enero de 1996 y mayo de 1997; se incluyó la descripción de las infecciones intrahospitalarias más frecuentes, sus tasas de incidencia y los agentes causales cuando fue posible establecerlos. Asimismo, se abordaron aspectos como la mortalidad relacionada y los costos generados por la sobrestancia hospitalaria de los pacientes infectados.

El análisis de los datos se realizó con la prueba $c^{2}$ para datos no paramétricos y con $t$ de Student para datos paramétricos.

\section{Resultados}

Durante el periodo de estudio se produjeron 10203 egresos, con un promedio mensual de 600.17 (rango 429-678). Se captaron 509 casos de infección nosocomial, para una tasa general de infecciones por 100 egresos de $4.99 \%$; las tasas notificadas mensualmente oscilaron entre 7.06 y 2.73 , con variaciones importantes relacionadas, sobre todo, con los periodos vacacionales tanto del coordinador del comité como del encargado de la vigilancia:

La tasa de infecciones, cuando estuvo ausente el coordinador, fue de 3.44 vs 5.34 al estar él presente $\left(c^{2}\right.$ $p<0.001$, RM 0.63, IC95\% 0.83-0.48).

La tasa de infecciones, en ausencia del encargado de la vigilancia, fue de 3.98 vs 4.99 mientras él se mantenía laborando ( $c^{2} p=0.01$, RM 0.74, IC95\% 0.58-0.93).

La tasa en ausencia de cualquiera de los dos fue de 3.81 vs 5.65 en presencia de ambos $\left(c^{2} p<0.001\right.$, RM 0.66 , IC95\% 0.81-0.54).

La tasa de incidencia de infección nosocomial fue de 4.34 (443 pacientes) con una fluctuación de entre 5.7 y 2.52. Se encontraron 1.15 infecciones por cada paciente infectado.

La tasa de infección por servicios fue como sigue: cirugía cardiotorácica, 16.8; neumología pediátrica, 8.2; cardiología pediátrica, 6.46; cardiología en adultos, 3.01; neumología en adultos, 2.9, y angiología, 1.13.

En las unidades de terapia intensiva las tasas de infección correspondieron a: unidad de terapia respira- toria, 14.55; unidad de terapia intensiva neonatal, 13.04; unidad de terapia posquirúrgica pediátrica, 10.49; unidad de terapia posquirúrgica adultos, 7.69 , y unidad de cuidados coronarios, 3.08 .

Respecto a su frecuencia, los de herida quirúrgica (IHQ) tuvieron una tasa de $1.14(\mathrm{n}=116)$; las de vías urinarias (IVU), de $1.08(\mathrm{n}=110)$; las bacteriemias primarias (BP), de $0.72(\mathrm{n}=74)$, y las neumonías nosocomiales $(\mathrm{NN})$, de $0.7(\mathrm{n}=71)$.

En lo concerniente a las infecciones relacionadas con catéteres intravasculares (ICI), se observaron 32 casos de flebitis (18.08\%), 59 de infección por catéter $(33.3 \%), 12$ bacteriemias por catéter $(6.78 \%)$ y $74 \mathrm{BP}$ $(41.8 \%)$. Los gérmenes encontrados más a menudo en BP $(n=94)$ fueron Staphylococcus coagulasa negativo $(24.5 \%)$, Candida spp. $(23.4 \%)$, Staphylococcous aureus $(13.08 \%)$, enterobacterias $(19.15 \%)$ y bacilos gramnegativos no fermentadores (9.6\%). Excluyendo a los hongos, las bacterias grampositivas fueron las causantes de $61.1 \%$ de las BP, y las gramnegativas, de $38.9 \%$. Las tasas específicas de ICI (incluyendo BP) por cada 100 días de utilización de catéteres centrales fue de 1.44; la tasa de ICI por cada 100 catéteres instalados fue de 27.4; el promedio de días que permanecía instalado un catéter central fue de 10.8. Al realizar una gráfica de las tasas mensuales de ICI, a pesar de que no se detectó un aumento importante en la tasa por 100 egresos, el incremento por tasas específicas fue mucho más evidente (figura 1).

Las IHQ ocuparon el primer lugar en frecuencia (1.14 por 100 egresados), con una tasa específica de IHQ por cada 100 cirugías de 4.6 (2 510 cirugías). Los gér-

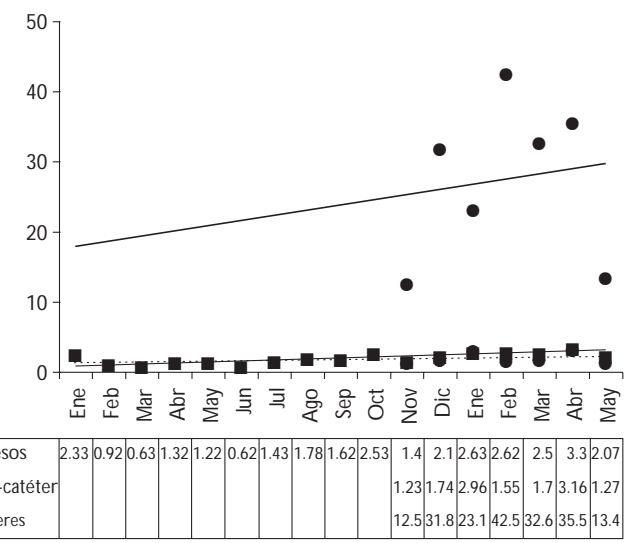

Figura 1. TASa MENSUAL de bacteriemias e INfeCción relacionada con catéter. Hospital de En fermedades Cardiovasculares y del Tórax No. 34, Instituto Mexicano del Seguro Social, Monterrey, Nuevo LEón, México, 1996-1997 
menes más frecuentemente aislados en IHQ $(n=54)$ fueron enterobacterias $(44.4 \%)$, bacilos gramnegativos no fermentadores $(22.2 \%)$ y S. aureus $(20.4 \%)$. Los gramnegativos causaron $66.7 \%$ de las IHQ, mientras que los grampositivos ocasionaron $33.3 \%$ de estas infecciones. Las IHQ se han mantenido estables en cuanto a su incidencia mensual por cada 100 egresos, pero han aumentado al calcularlas por cada 100 cirugías realizadas (figura 2).

De las IVU, $96.4 \%$ se relacionaron con cateterismo uretral. Los gérmenes más frecuentemente encontrados $(n=74)$ en este tipo de infecciones fueron Candida spp. (60.8\%) y enterobacterias (32.4\%). La tasa de IVU por cada 100 días de permanencia de la sonda uretral fue de 1.27, mientras que la tasa de IVU por cada 100 sondas instaladas fue de 16.5; el promedio de días de utilización de la sonda fue de 9.23. A pesar de no demostrarse un incremento significativo en la tasa de IVU al calcularla por 100 egresos, es evidente la tendencia al aumento al calcularlas tanto por cada 100 días de permanencia de la sonda uretral, como sobre la base del número de sondas instaladas mensualmente (figura 3).

Las NN estuvieron asociadas con la ventilación mecánica en $91.5 \%$ de los casos. Los gérmenes más frecuentemente vinculados a $\mathrm{NN}(\mathrm{n}=36)$ fueron: bacilos gramnegativos no fermentadores en $50 \%$ y enterobacterias en $41.7 \%$. Las tasas específicas de NN se dieron como sigue: por 100 días de ventilación mecánica, 1.07, y por cada 100 pacientes intubados, 8.14; el promedio de días en ventilación mecánica por paciente fue de 7.7 días. Al realizar una gráfica de la tendencia de este tipo de infecciones, se encontró una disminución pro-

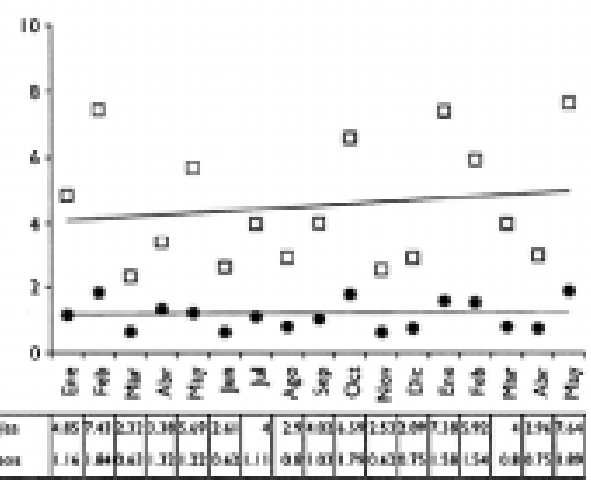

Figura 2. TASA MENSUAL de INFECCIÓN DE heRIDA QUIrúrgica. Hospital de Enfermedades Cardiovasculares y del Tórax No. 34, Instituto Mexicano del Seguro Social, Monterrey, Nuevo león, México, 1996-1997 gresiva en las tasas específicas, mas no en la tasa por 100 egresos (figura 4).

Por otra parte, el promedio de días de estancia de los pacientes infectados fue significativamente mayor en comparación con el de los pacientes no infectados: 20.68 vs 8.54, respectivamente $(p<0.001)$. Esa sobrestancia prácticamente triplicada, se tradujo en un costo extra promedio diario de 17446.64 pesos por cada paciente infectado.

Se encontró que de los 443 pacientes infectados, 96 fallecieron $(21.7 \%)$, cifra que representa $0.94 \%$ de
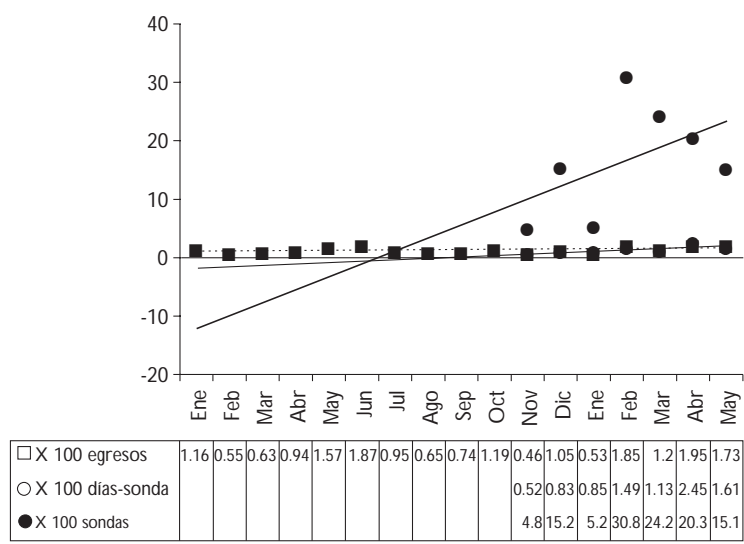

Figura 3. Tasa mensual de infección urinaria. HosPITAL de En fermedades Cardiovasculares y del TóRaX No. 34, Instituto MExicano del Seguro Social, Monterrey, Nuevo León, México, 1996-1997

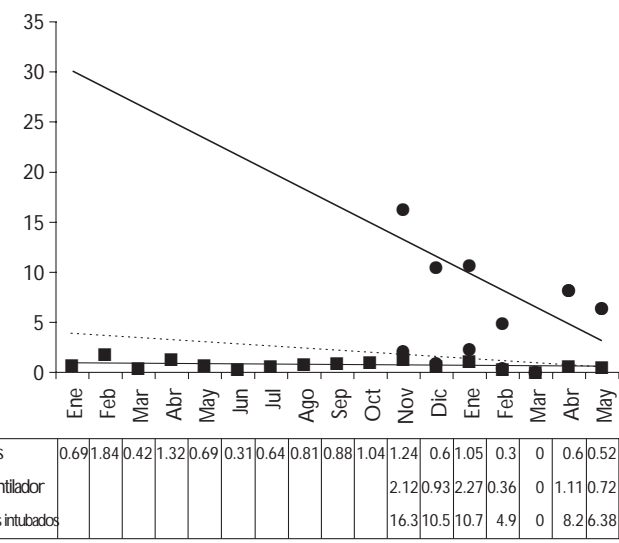

Figura 4. Tasa mensual de neumonías nosocomiales. Hospital de En fermedades Cardiova sculares y del Tórax No. 34, Instituto Mexicano del Seguro Social, Monterrey, Nuevo León, México, 1996-1997 
todos los egresos. De la cifra de decesos, $36.5 \%$ estuvieron relacionados directamente con la infección. $\mathrm{Al}$ analizarla con base en el tipo de infección, la mortalidad mayor estuvo asociada con las BP (36.5\%) y con las NN (34.3\%).

\section{Discusión}

Las tasas de infección nosocomial del HECVT 34 fueron más bajas que las notificadas por el Instituto Nacional de Cardiología en $1985^{2}$ y que las de Toronto en $1988,{ }^{3}$ probablemente por una mayor cantidad de pacientes en este estudio. Sin embargo, también es posible que haya subregistro en las cifras de este estudio, sobre todo considerando las grandes diferencias encontradas con la ausencia del personal habitualmente encargado de la vigilancia. Esto ocurre a pesar de que el Departamento de Personal realiza una cobertura rutinaria durante los periodos vacacionales. El análisis de estos datos es importante ya que refleja un problema cotidiano en las instituciones de salud en México, donde frecuentemente ocurren eventualidades que ponen en riesgo una vigilancia continua. Es necesario analizar la necesidad de aumentar el personal dedicado a la vigilancia, así como la capacitación de las personas encargadas de desempeñar las tareas del personal especializado cuando éste se encuentra ausente.

Otra posible explicación de la disparidad entre los informes sería la metodología utilizada en la captación de los casos, sobre todo en lo que corresponde al Instituto Nacional de Cardiología de México, ${ }^{2}$ donde se incluyen fiebre de origen probablemente infeccioso y fiebre postoperatoria, las cuales no se consideraron en los casos de este estudio. Las diferencias entre tasas de infección nosocomial no son únicas en este caso, sino que ocurren frecuentemente inclusive en informes de hospitales de México y del extranjero. Aunque se utilizaron metodologías diferentes -lo que no permite hacer comparaciones-, las tasas de infección nosocomial encontradas en cardiología obligan a hacer especial énfasis en la vigilancia y el control de las infecciones nosocomiales en estas áreas consideradas de menor riesgo.

Las salas de terapia intensiva fueron las que presentaron mayores tasas de infección, especialmente aquellas donde se manejan pacientes con patología respiratoria crónica y que, por ende, requieren de apoyo ventilatorio mecánico durante más tiempo, y aquellas donde se atiende a pacientes postoperados de pediatría, tanto neonatos como lactantes y prescolares. En estas áreas se utilizó la mayor parte de los antibióticos, especialmente los de amplio espectro, y se aisló a la mayoría de los organismos multirresistentes y de las levaduras.
A diferencia de lo que ocurre en otro tipo de hospitales en los cuales las IVU son las más frecuentes, ${ }^{6}$ en el HECVT 34 -donde en general los pacientes presentan problemas cardiovasculares y no reciben medicamentos citotóxicos, pero sí son objeto de un gran número de procedimientos invasivos vasculares (cateterismos, monitorización invasiva, colocación de marcapasos, prótesis intravasculares, etc.)- la mayor parte de las infecciones registradas son intravasculares, siendo las BP las de mayor gravedad por su alta mortalidad y sus complicaciones. Los pacientes más afectados por este tipo de infecciones fueron los postoperados, especialmente en pediatría. Por tratarse de pacientes cardiópatas y debido a que los organismos grampositivos y las levaduras constituyen la generalidad de los patógenos asociados a bacteriemias, siempre está presente la posibilidad de que se desarrolle una endocarditis, de tal manera que en todos los casos de bacteriemia se realizó una búsqueda exhaustiva de esta complicación infecciosa, que ocurrió en cinco ca$\operatorname{sos}(0.67 \%)$.

Las IHQ ocupan el primer sitio entre las infecciones nosocomiales debido al gran número de cirugías que se practican; se ha encontrado que su número ha aumentado especialmente a raíz de la inclusión de los servicios de pediatría en el hospital. La mayor dificultad técnica que la cirugía de cardiopatías congénitas conlleva, así como el mayor riesgo de infección que corren los cardiópatas -por la cronicidad de su padecimiento, por su alta frecuencia de malnutrición y por su corta edad, especialmente en el periodo neonatal- se cuentan como causas de una mayor morbilidad en este grupo de edad. Se puede apreciar que en el Hospital for Sick Children ${ }^{3}$ la cuarta parte de los pacientes pediátricos sometidos a cirugía presenta infección de herida quirúrgica. Otros problemas asociados al incremento de las infecciones de herida son la diabetes mellitus, la obesidad, los problemas circulatorios crónicos, el sangrado postoperatorio abundante y el tiempo largo de cirugía, que a menudo se presentan entre los pacientes quirúrgicos adultos del HECVT 34. De acuerdo con el Sistema Nacional de Vigilancia de Infecciones Nosocomiales de EUA, la tasa promedio de infección de herida quirúrgica más alta ocurre en cirugía cardiovascular, con 2.5 por cada 100 egresos; ${ }^{7}$ esa cifra duplica a la encontrada en el HECVT 34 (1.4 por 100 egresos), lo cual probablemente refleja el hecho de que la mayoría de las cirugías que ahí se practican son de tipo electivo. En general, la tasa específica de IHQ en este hospital (4.6 por 100 cirugías) también refleja la baja incidencia de este problema entre los pacientes. Otra razón para las bajas tasas de IHQ pudiera ser la puesta en marcha, desde un ini- 
cio, de un programa estricto de profilaxis antimicrobiana en cirugía cardiovascular, en el cual se recurre a la monoterapia con betalactámicos de acción preponderante contra grampositivos, y no a esquemas con mayor actividad contra gramnegativos. ${ }^{8}$ Este protocolo también ha dado como resultado una mayor tasa de recuperación de gramnegativos en IHQ, aunque éstos en rara ocasión son resistentes a aminoglucósidos y a cefalosporinas de tercera generación, a diferencia de lo que ocurre en los aislados de muestras de secreción respiratoria.

Las IVU representan un problema de consideración, pues a pesar de que sólo los pacientes quirúrgicos y los severamente enfermos de las terapias intensivas son sometidos a cateterismo vesical, este tipo de infecciones presenta una tendencia al incremento, especialmente si se parte del número de sondas uretrales colocadas. Sin lugar a dudas es prioritaria una revisión de la técnica de instalación y mantenimiento de dichos dispositivos en el HECVT 34.

El gran logro del programa de control de infecciones lo constituye la tasa de NN que, gracias a la vigilancia de las técnicas de aseo bronquial y mantenimiento de la vía aérea, así como al programa de extubación temprana sobre todo en los adultos, muestra una disminución paulatina en su incidencia. Las tasas tan bajas son todavía más significativas por tratarse de un hospital de patología torácica.

Cabe destacar aquí la relevancia que tiene el análisis de las tasas específicas por procedimientos o dispositivos, ya que en todos los tipos de infección nosocomial, la tasa por 100 egresos no reflejó de manera importante las tendencias en cuanto a la incidencia mensual de las infecciones. En otras palabras, si se calcula exclusivamente la tasa por 100 egresos de cada tipo de infección, es difícil establecer la problemática que ese tipo particular de infección representa para el hospital, ya que no todos los pacientes se encuentran sujetos a los mismos riesgos.

La sobrestancia hospitalaria de los pacientes infectados siempre será relevante al realizar cualquier análisis acerca del costo que implica mantener un programa de vigilancia y control de infecciones. En este estudio, la estancia hospitalaria de un paciente infectado se triplica en comparación con la de un enfermo que no presenta esa complicación. Si dicha circunstancia pudiera evitarse, al menos parcialmente, cabría la posibilidad de internar cuando menos a otro paciente en sustitución de aquel que tuvo complicaciones, con lo cual se aprovecharía mejor la capacidad hospitalaria tan saturada hoy en día, además de que un paciente no complicado requiere de menos atención especializada, así como de una menor cantidad de medicamentos y de procedimientos caros y riesgosos.

En el HECVT 34 el único costo que se puede atribuir al programa de vigilancia son los salarios del coordinador y del encargado de la vigilancia, ya que ambos se dedican a esta labor de manera prácticamente exclusiva, sacrificando otras actividades que requieren de personal extra. Este costo es sumamente reducido si se considera que por cada día de sobrestancia de cada paciente infectado (sin considerar medicamentos o procedimientos extra), se gasta una cantidad mayor que el salario de ambos trabajadores en un mes.

Las cifras que se presentan aquí, constituyen una base sobre la cual trabajar en los próximos años; evidentemente falta mucho por hacer, sobre todo en relación con la prevención de ICI e IVU, pero existe un compromiso serio por parte de las autoridades para apoyar las medidas preventivas y educativas a fin de reducir al mínimo las tasas de infección intrahospitalaria y compartir estas experiencias con el resto de los hospitales de la delegación norte del IMSS.

\section{Referencias}

1. Ponce de León-Rosales Samuel, Ponce de León-Rosales Sergio, RuizPalacios G, Gutiérrez R. Infecciones nosocomiales: características del problema en el Instituto $\mathrm{N}$ acional de la N utrición Salvador Zubirán y en México. Salud Publica Mex 1986;28:29-36.

2. Ponce de León-Rosales S, G arcía-G arcía ML,Volkow-Fernández F. Resultados iniciales de un programa de vigilancia de infecciones nosocomiales en los Institutos N acionales de Salud. Salud Publica Mex 1986;28:583-592. 3. Pollock EMM, Ford-Jones EL, Rebeyka L, Mindorff CM, Bohn DJ, Edmonds JF et al. Early nosocomial infections in pediatric cardiovascular surgery patients. Crit Care Med 1990;18: 378-384.

4. Garner JS, Jarvis W R, Emori TG, Horan TC, Hughes JM. CDC definitions for nosocomial infections, 1988. Am J Infect Control 1988;16:128-140.

5. Wenzel RP, 0 sterman CA, Hunting KJ. Hospital acquired infections: II Infections by site, service and common procedure in a university hospital. Am J Epidemiol 1978;104:645-651.

6. Haley RW, Culver DH, W hite JW, Morgan W M, Emori TG. The nationwide nosocomial infection rate:A new need for vital statistics. Am J Epidemiol 1985;121:159-167.

7. Anonymous. Nosocomial infection rates for interhospital comparison: Limitations and possible solutions. Infect Control Hosp Epidemiol 1991;12:609-621.

8. Miedzinski LJ, C allaghan JC, Fanning EA, G elfand ET, G oldsond G, Mondry $D$ et al.Antimicrobial prophylaxis for open heart operations.Ann Thorac Surg 1990;50:800-807. 